\title{
ESTUDO DO COMPORTAMENTO TÉRMICO DE MOTOR A COMBUSTÃO INTERNA SUBMETIDO A DESBALANCEAMENTO EM DIFERENTES POSIÇÕES
}

\author{
Marcel Henrique Lopes ${ }^{(1)}$ (marcel.henrike@ @otmail.com), Elen Kássia de Assis ${ }^{(1)}$ \\ (elen.kassia@engenharia.ufjf.br), Jorge Nei Brito ${ }^{(2)}$ (brito@ufsj.edu.br), José Antônio da Silva ${ }^{(3)}$ \\ (jant@ufsj.edu.br) \\ (1) Univ. Federal de São João Del Rei (UFSJ) - Grupo de Estudos e Pesquisas do Laboratório de Sistemas Dinâmicos (Gep_LASID) \\ (2) Universidade Federal de São João Del Rei (UFSJ) - Departamento de Engenharia Mecânica (DEMEC) \\ (3) Universidade Federal de São João Del Rei (UFSJ) - Departamento de Ciências Térmicas e dos Fluidos (DCTEF)
}

RESUMO: $O$ desbalanceamento é um fator que ocorre com frequência em equipamentos e conhecer o modo como ele se apresenta em espectros vibracionais e termogramas é de suma importância para identificar possíveis falhas. A Termografia, técnica de análise e caracterização empregada neste trabalho, é uma técnica de sensoriamento remoto que permite a medição de temperaturas $e$ formação de imagens térmicas de equipamentos elou processos, a partir da radiação infravermelha emitida naturalmente pelos corpos. Em motores elétricos, a forma como os modos de falha aparecem nos espectros é conhecido e consolidado. Entretanto, para motores a combustão interna, este tipo de análise não é tão simples, fazendo-se necessário adotar métodos que permitam caracterizar de forma não invasiva os possíveis modos de falha. Para este estudo, previamente foi definida a "Assinatura de Vibração" do motor, que é a frequência vibracional deste em condições ideais de funcionamento e seu respectivo termograma, para que os demais fossem baseados nele. Em seguida, foram inseridas as massas de desbalanceamento para que o modo operante com este tipo de falha fosse caracterizado, permitindo assim que, caso ocorra o desbalanceamento, este possa ser identificado por meio dos dados vibracionais do motor e seus respectivos termogramas. Os dados foram coletados em três direções, vertical, horizontal e axial, por meio de acelerômetros piezelétricos que transmitiram o sinal para o equipamento de monitoramento on-line IMx-S 16 da SKF. A posição vertical foi a que apresentou resultados mais interessantes para análise. Os dados foram armazenados e tratados no software @ ptitude Analyst, também da SKF. As imagens térmicas foram obtidas por meio da câmera T200 da FLIR e posteriormente tratadas no software FLIR Quick Report.

PALAVRAS-CHAVE: Manutenção preditiva. Termografia. Vibração. Motor a combustão interna. Desbalanceamento.

\section{INTRODUÇÃO}

Manutenção preditiva é definida como a manutenção que permite garantir uma qualidade de serviço desejada, com base na aplicação de técnicas de análise, utilizando-se meios de supervisão ou de amostragem (Oliveira, 2012). 

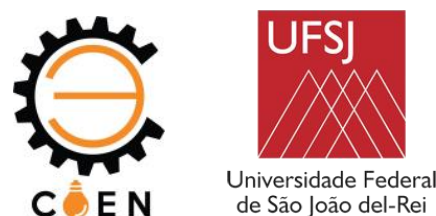

Através de técnicas preditivas é feito o monitoramento da condição e a ação, quando necessária, é realizada através de manutenção corretiva planejada.

A termografia é a técnica preditiva que permite o acompanhamento da temperatura e a formação de imagens térmicas, denominadas termogramas. A temperatura é um dos parâmetros de mais fácil compreensão e o acompanhamento de sua variação é muito importante tanto para equipamentos elétricos como mecânicos, pois permite constatar alterações na condição de componentes e do próprio processo (Souza, 2011).

A técnica de análise termográfica consiste na obtenção de imagens que são geradas através do diferencial de temperatura emitido pela radiação dos corpos.

De Acordo com Bassi (2012), a radiação térmica é emitida continuamente pelos materiais devido à agitação atômica e molecular associada com a energia interna dos mesmos. Na termografia são usados detectores de radiação para monitorar a energia de radiação térmica de uma superfície na porção infravermelha do espectro e avaliar sua temperatura.

Segundo Dionísio (2017), todos os objetos emitem radiação infravermelha, sendo que a intensidade da radiação emitida depende da temperatura do objeto e da capacidade de o objeto emitir radiação, conhecida por emissividade $(\varepsilon)$. A emissividade é definida como a capacidade que o corpo dispõe para irradiar energia quando comparada com a de um corpo negro perfeito. Um corpo negro perfeito é aquele que absorve toda a radiação eletromagnética que nele incide.

A técnica termográfica mais usada na indústria é a comparação termográfica, sendo que esta comparação pode ser quantitativa ou qualitativa. A termografia quantitativa é um método utilizado para avaliar a condição do equipamento, através da comparação dos valores de temperatura com equipamentos idênticos, valores de referência ou limites. O método de comparação qualitativo é idêntico ao anterior. Contudo, ao invés de utilizar a comparação de valores utiliza uma imagem padrão termográfica. Embora as medições qualitativas possam detectar deficiências, é através das medições quantitativas que se tem a capacidade para determinar o potencial de gravidade da falha (Campos, 2011).

Bassi (2012), afirma que no interior dos cilindros de um motor de combustão interna é que ocorrem complexos fenômenos de transformação de energia, principalmente pelo processo de combustão. Durante os processos que ocorrem no cilindro de um motor, o fluido de trabalho sofre grandes variações de temperatura e pressão. Essas variações associadas à natureza turbulenta, transitória e tridimensional do escoamento determinam o comportamento da troca de calor entre o fluido de trabalho e paredes do cilindro, do pistão e do cabeçote.

Os compostos gerados pela combustão também participam dos processos de trocas térmicas internas no motor. Gases com moléculas assimétricas como dióxido de carbono (CO2), monóxido de carbono $(\mathrm{CO})$, dióxido de enxofre (SO2), água (H2O) e hidrocarbonetos participam do processo de transmissão de calor por absorção de radiação a temperaturas moderadas e por absorção e emissão de radiação a altas temperaturas, como ocorre nos cilindros dos motores de combustão interna (Çengel, 2002).

O motor de combustão interna é um conversor de energia química em energia mecânica. Sabese que na prática os conversores de energia não são perfeitos, portanto, não são capazes de converter totalmente a forma de energia na entrada do sistema para a forma de energia na saída. As perdas energéticas em um motor de combustão interna ocorrem pelo calor rejeitado nos gases de exaustão, combustão incompleta, atrito entre partes móveis, calor rejeitado pelo fluido de arrefecimento, calor rejeitado pelo fluido lubrificante e calor rejeitado ao ambiente pelas superfícies externas do motor. 

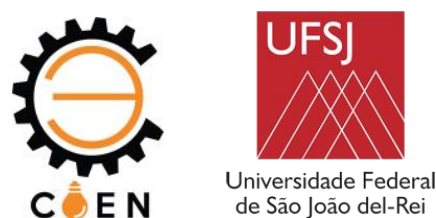

Quase um terço da energia do combustível deve ser dissipado ao ambiente de alguma forma, seja pelo sistema de arrefecimento e óleo lubrificante ou pelas superfícies externas do motor (Bassi, 2012).

Segundo Bassi (2012), a transferência de calor nos motores afeta seu desempenho, eficiência e emissões. Se grande quantidade de calor é transferida para as paredes da câmara de combustão a temperatura e a pressão média dos gases diminuem, reduzindo o trabalho por ciclo transferido para o cilindro.

Em razão das altas temperaturas na câmara de combustão podem ocorrer algumas falhas que reduzirão a vida útil do motor, como autoignição, pré-ignição e detonação. O efeito autoignição ocorre quando pontos quentes no interior da câmara passam a fazer o papel da vela de ignição, incandescendo a mistura ar/combustível antes mesmo da vela de ignição iniciar o processo através da centelha elétrica. Este efeito é muito prejudicial ao funcionamento do motor, fazendo com que o mesmo perca potência e corra o risco de superaquecimento. A autoignição pode levar à destruição da câmara de combustão e, em casos extremos, furos na cabeça dos pistões ou mesmo sua fusão com o cilindro. A pré-ignição provoca a queima da mistura antes do tempo normal de combustão, ao contrário da detonação que a atrasa. A detonação pode ser definida como uma combustão proveniente da reação rápida e espontânea de uma parte da mistura ar/combustível, quando esta é submetida a pressões e temperaturas crescentes originadas da combustão normal. A detonação cria uma explosão com pressão e velocidades violentas dentro da câmara, como o motor não pode efetivamente utilizar esta energia, ela é dissipada na forma de calor e vibração de alta frequência, que podem exercer esforços sobre os pistões e anéis além dos seus limites de resistência mecânica (Malpica, 2007).

De acordo com Rezende (1991), a análise de vibração tem o intuito de diagnosticar um problema mecânico e sua origem, a partir de um quadro de sintomas determinado pelas características da vibração. A análise por assinatura de vibração é um dos métodos mais aplicados atualmente na identificação de falhas. A sua premissa básica é de que existem certas características espectrais que, em termos gerais, identificam determinados tipos de defeitos.

O motor é a principal fonte de vibração do veículo. As vibrações totais provenientes do motor podem ser divididas em dois grupos: vibração devido ao processo de combustão e vibração devido às forças mecânicas (válvulas e pistões), sendo a primeira mais influente na vibração global do motor.

A Assinatura de Vibração pode ser definida como a frequência padrão vibracional do motor, desde que ele esteja em condições ideais de funcionamento e seus componentes e respectivas frequências naturais sejam conhecidas.

De acordo com Golwine et al. (2004), a análise da assinatura de vibração no bloco do motor e a análise da variação de torque e potência do motor estão entre as principais abordagens sugeridas para se medir a qualidade da combustão entre os diferentes cilindros do motor. Também de acordo com Golwine et al. (2004), informações sobre a qualidade da combustão em um motor de combustão interna proporcionam uma forte ferramenta de diagnóstico no que diz respeito à operação do motor e sua saúde.

Com a assinatura de vibração pode-se monitorar as condições de uma máquina, tendo esta como base a realização da manutenção em indicadores de desgastes ou falhas. Sendo isso possível através da identificação de padrões para os sinais quando a máquina está operando dentro de suas condições ideias, monitorando-se então estes sinais para que, quando os mesmos se distanciem dos padrões, indicando desgaste ou proximidade de alguma falha, se realize a manutenção (Prado, 2003).

Brito (2002) destaca que o desbalanceamento de rotores é, sem dúvida, a maior causa de vibrações em máquinas rotativas. Este fenômeno é caracterizado pela existência de desequilíbrios de 
massa em relação aos eixos de rotação. A vibração resultante é predominantemente radial, forte componente em uma vez a frequência de rotação $(1 x f r)$.

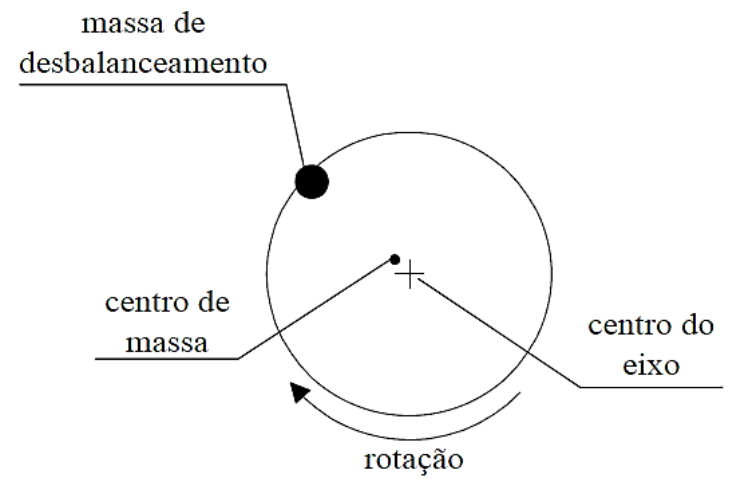

Figura 1. Desequilíbrio de massa. Fonte: Brito (2002).

Baccarini (2005) explica que o desbalanceamento gera uma força que é estacionária em relação ao rotor. O sinal no tempo é senoidal e cada ciclo tem a mesma amplitude e depende da quantidade de desbalanceamento. Assim, a vibração predominante ocorre na frequência de rotação do rotor e terá amplitudes maiores nas direções radiais (horizontal e vertical).

$\mathrm{O}$ motor em estudo, possui um sistema de arrefecimento a ar. Este possui um volante que gera um fluxo de ar para as aletas presentes no cilindro do motor, aumentando o fluxo de acordo com o aumento da rotação.

\section{MATERIAIS E MÉTODOS}

\subsection{Motor de Estudo}

Os testes foram realizados no Laboratório de Energia do Departamento de Ciências Térmicas e dos Fluidos - DCTEF da Universidade Federal de São João Del Rei - UFSJ. Foi utilizada uma bancada dinamométrica Tecquipment - TQ, Figura 2, composta de um freio hidráulico modelo TD200, equipada com placa de aquisição de dados e instrumentação que permite com o uso dos softwares VDAS e ECA100, análise de torque, potência, pressão atmosférica e rotação do motor.

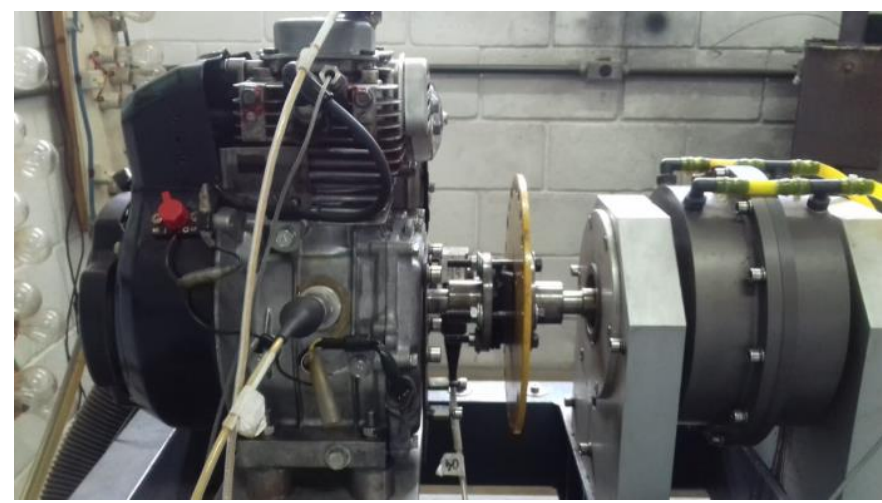

Figura 2. Bancada dinamométrica. Fonte: Autores. 
O motor de estudo é o motor a gasolina da marca Subaru modelo Robin EH17-2D, monocilindro, a gasolina, naturalmente aspirado e refrigerado a ar, mostrado na Figura 3.

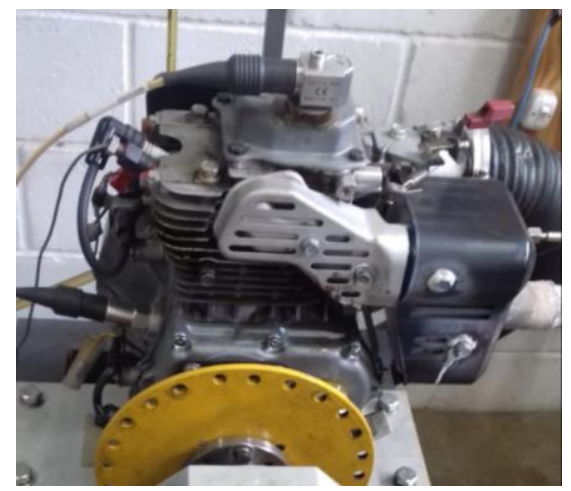

Figura 3. Direção axial do motor de estudo. Fonte: Autores.

\subsection{Câmera termográfica}

Para a captura das imagens foi utilizada a câmera termográfica Flir modelo T200, apresentada na Figura 4. A calibração da câmera foi feita com o auxílio de termômetro digital. Este foi posicionado no bloco do motor e a emissividade foi ajustada com base nas temperaturas identificadas. As imagens foram coletadas na direção axial a uma distância de 1 metro do motor a ser analisado.

Os termogramas foram tratados no software FLIR Quick Report para identificação dos pontos de interesse para análise e remoção das temperaturas da vizinhança do motor, pois estas não se aplicavam ao estudo.

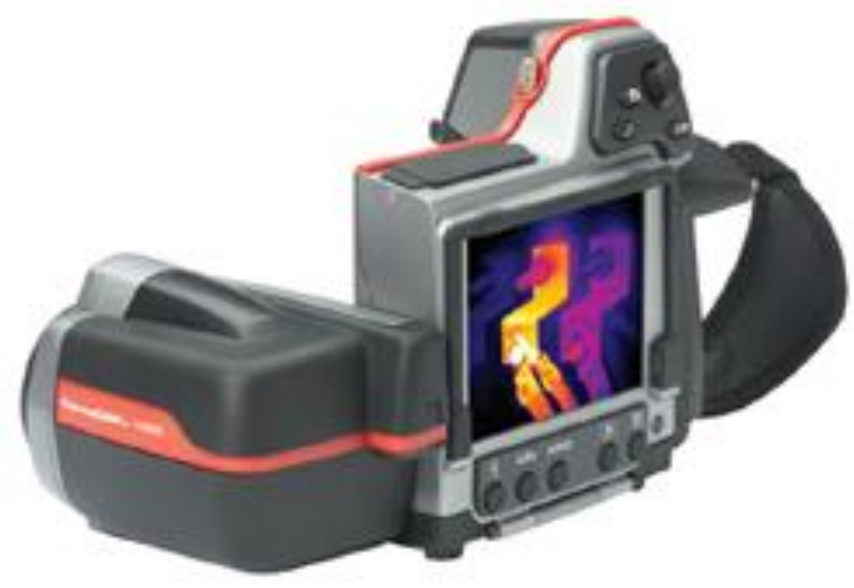

Figura 4. Câmera termográfica. Fonte: Manual do Fabricante FLIR. 


\subsection{IMx - $16 \mathrm{~S}$}

O IMX-S 16 é uma tecnologia SKF capaz de coletar dados através de diferentes sensores (acelerômetros, alicate amperímetro, bobina de fluxo magnético dentre outros) e enviar em tempo real para um banco de dados que pode ser acessado remotamente. $\mathrm{O}$ software de armazenamento permite que rotas de monitoramento sejam criadas e que os dados sejam analisados através de suas várias ferramentas disponíveis, o que facilita o diagnóstico dos problemas analisados. Para cada ponto de coleta de vibração podem ser gerados espectros de deslocamento, velocidade, aceleração e envelope simultaneamente.

Os sinais foram coletados por acelerômetros SKF CMSS2200 nas posições Vertical e Axial, e um acelerômetro SKF CMSS2100 na posição Horizontal.

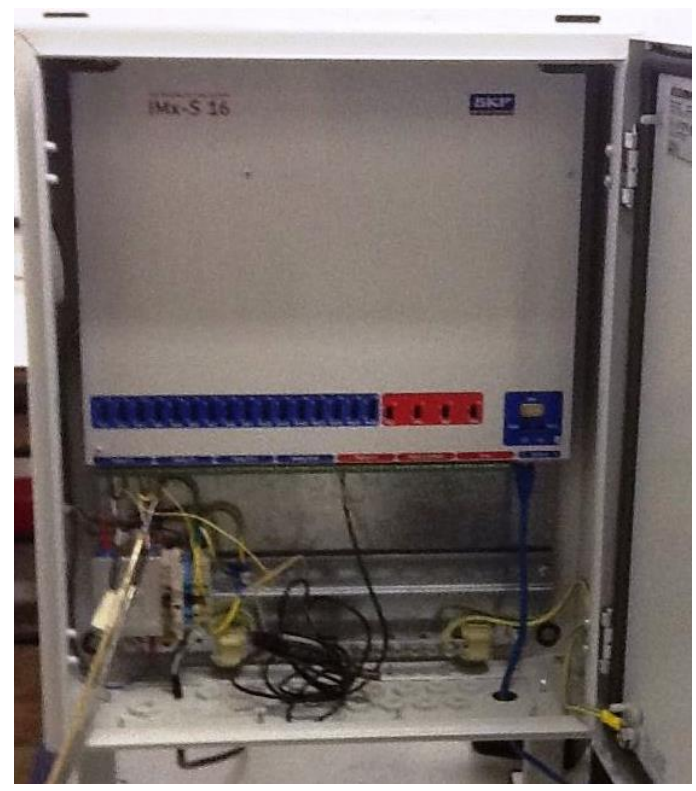

Figura 5. Sistema de aquisição de dados - IMx-S 16. Fonte: Autores.

\subsection{Software SKF @ptitude Analyst}

Primeiramente, elaborou-se um banco de dados contendo as faixas de frequência, unidades de medida, tipo de coleta, velocidade de trabalho e componentes relevantes para caracterização da frequência de vibração. A velocidade de trabalho foi fixada em 3000 RPM, a frequência limite adotada foi $5000 \mathrm{~Hz}$, o corte de frequência se deu em $6,25 \mathrm{~Hz}$ e o sinal representativo resulta de quatro médias.

Os sinais coletados foram aceleração, velocidade e envelope, sendo as duas primeiras por meio de detecção RMS e a última por pico. Conectado via ethernet ao IMx-S 16, o computador que abriga o software @ ptitude Analyst armazenou os dados provenientes dos acelerômetros, identificando a data e horário no qual o sinal foi coletado. O software permite a geração de espectros para melhor visualização das faixas vibracionais da máquina em estudo. 


\section{RESULTADOS E CONCLUSÕES}

Os testes foram realizados com a rotação mantida constante, a 3000 RPM, e o valor de torque aplicado foi $2 \mathrm{Nm}$, simulando algum tipo de carga (ou atrito). O disco foi desbalanceado com uma massa de $13,09 \mathrm{~g}$ em três posições diferentes do disco, sendo elas, $0^{\circ}, 180^{\circ}$ e $270^{\circ}$ (sentido horário) em relação ao pistão em Ponto Morto Superior (PMS).

O resfriamento do motor se dá por meio de um volante que gera uma corrente de ar contínua e proporcional à rotação. Entretanto, inserindo o desbalanceamento são geradas maiores forças vibratórias, aumentando também a sua temperatura. Conforme os espectros abaixo podemos observar a vibração de acordo com a posição do desbalanceamento.

A posição vertical foi a que apresentou resultados interessantes para este estudo, portanto, foi dada maior atenção a ela.

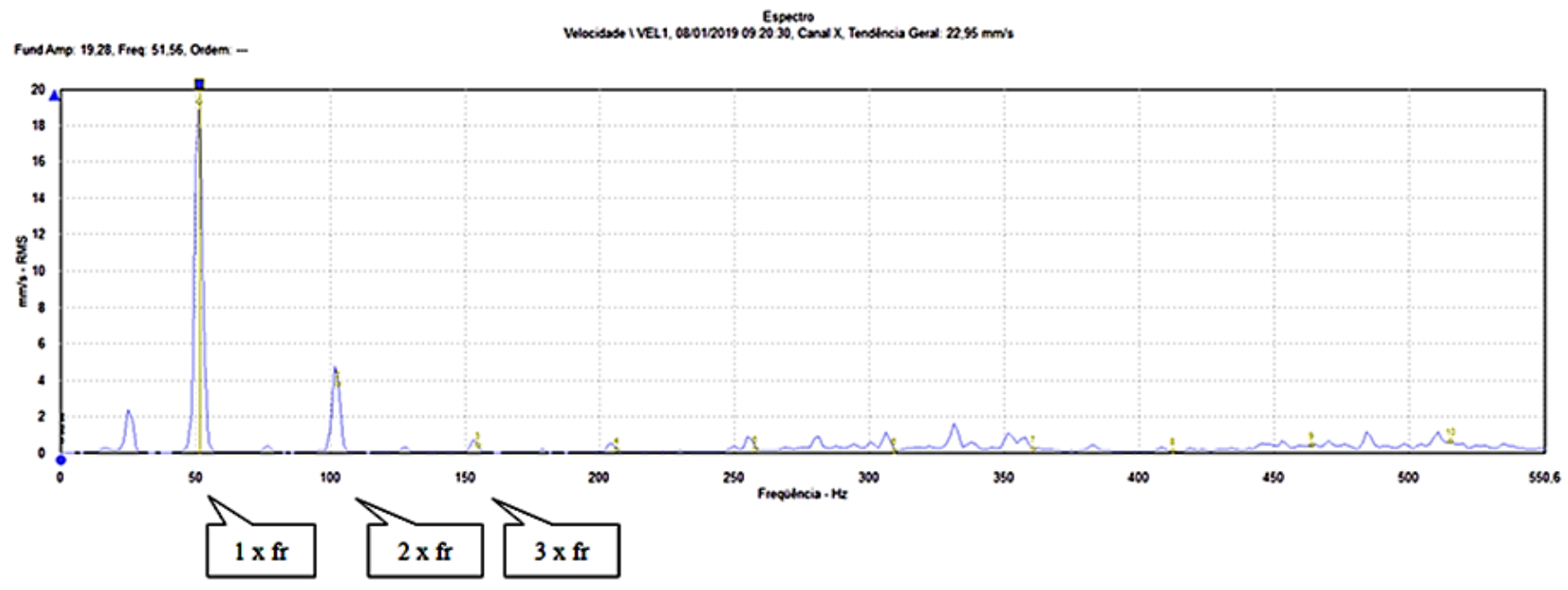

Figura 6. Assinatura vertical. Fonte Autores.

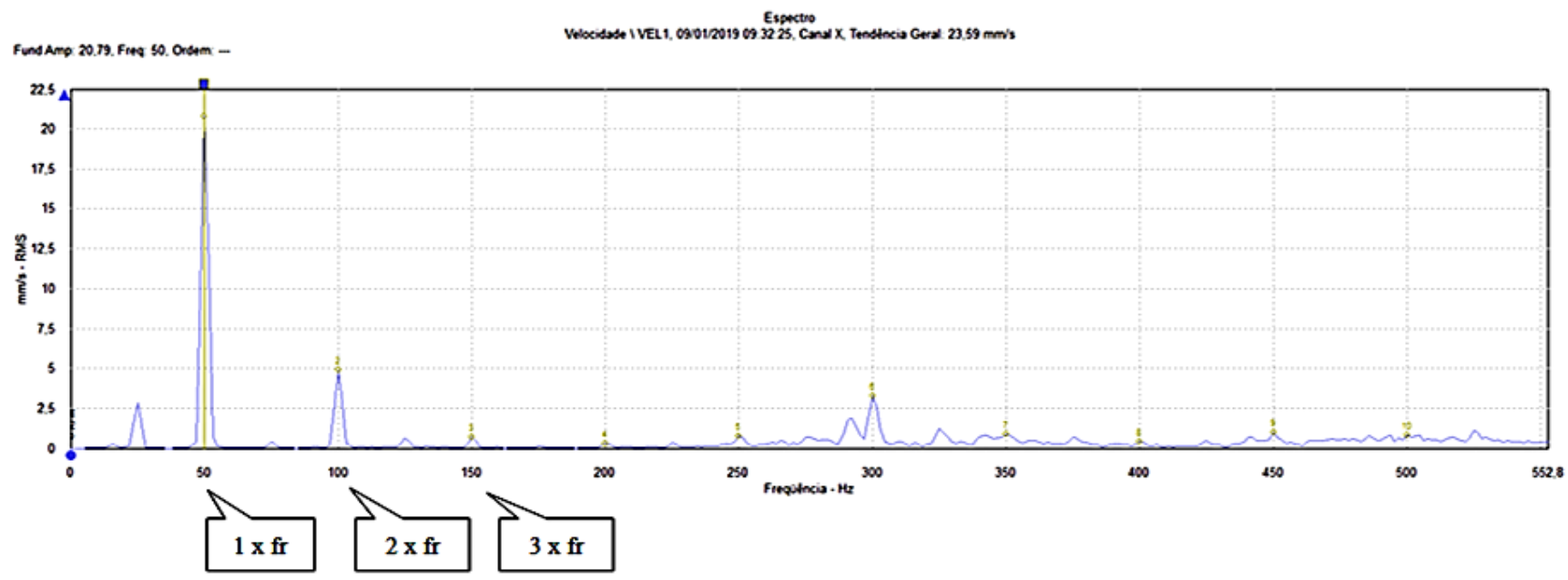

Figura 7. Desbalanceamento a $0^{\circ}$ do pistão em PMS. Fonte Autores. 


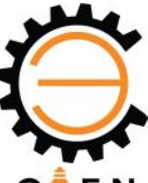

C $\mathrm{EN}$

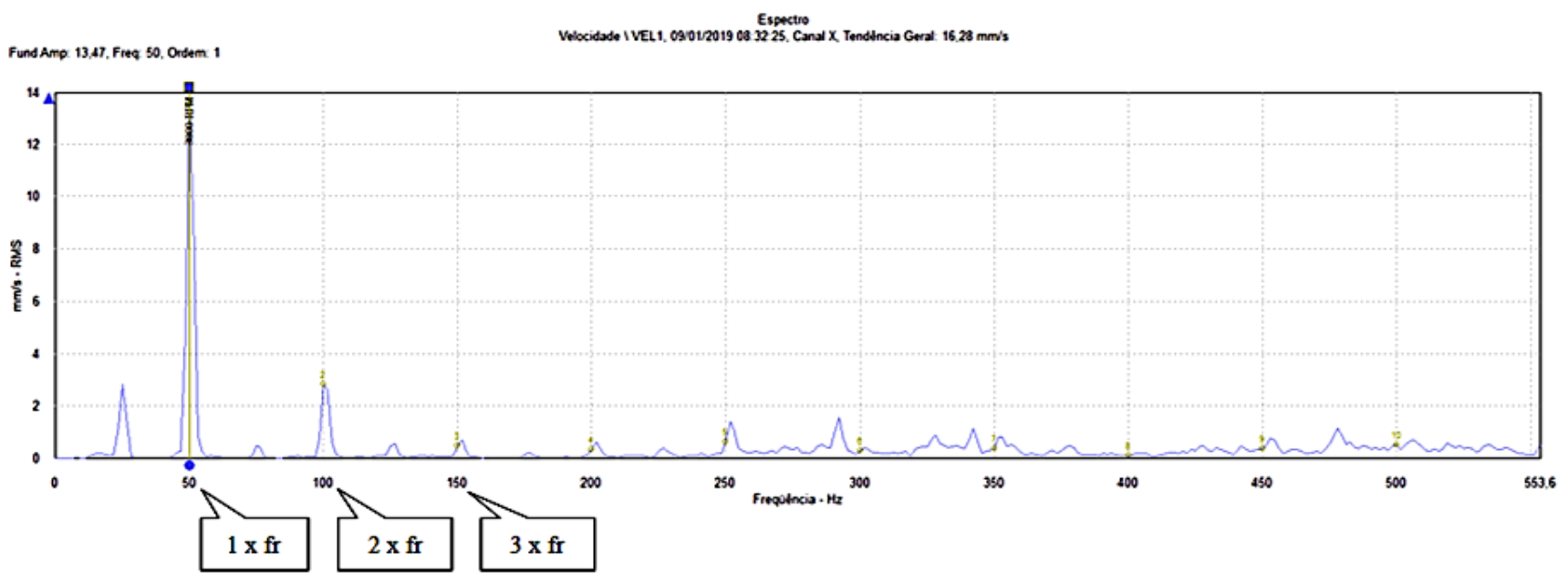

Figura 8. Desbalanceamento a $180^{\circ}$ do pistão em PMS. Fonte Autores.

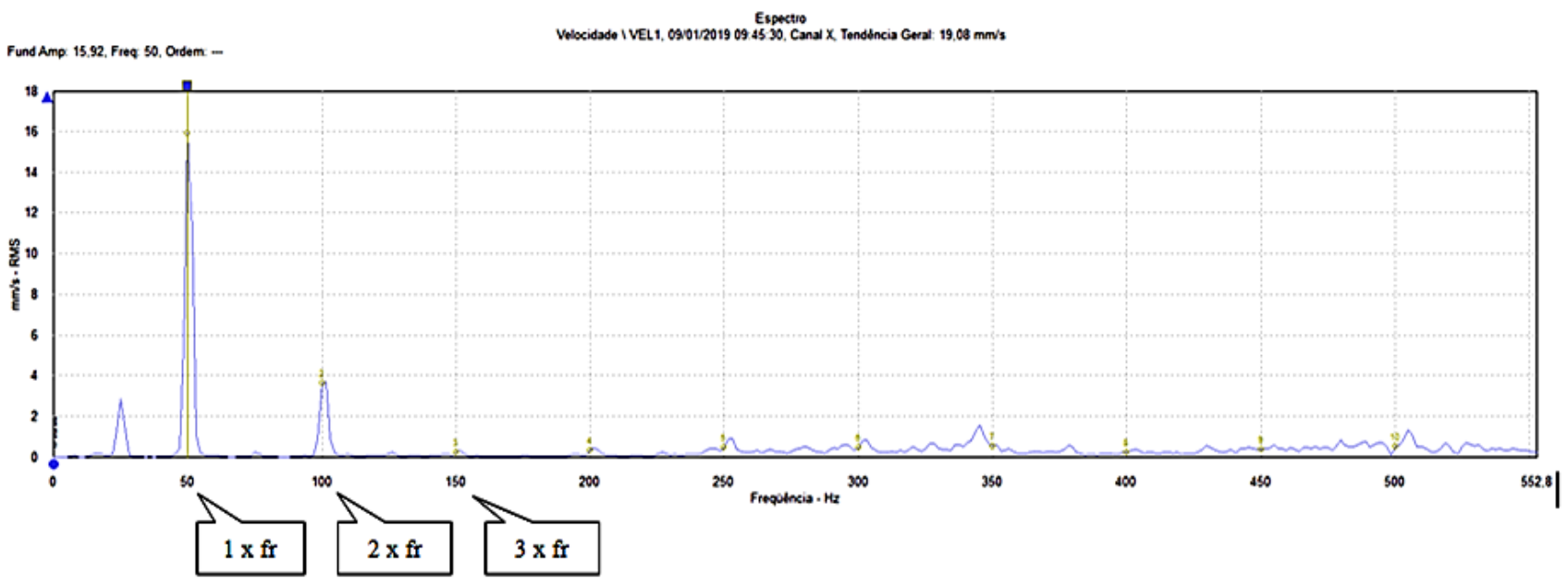

Figura 9. Desbalanceamento a $270^{\circ}$ do pistão em PMS. Fonte Autores.

Analisando os espectros gerados, pode-se perceber que a posição do desbalanceamento pode aumentar ou diminuir a vibração vertical. No presente estudo, a vibração aumenta quando o desbalanceamento está a $0^{\circ}$ e $270^{\circ}$ em relação ao PMS e diminui quando está a $180^{\circ}$, o que pode ser entendido levando em conta que o movimento do desbalanceamento pode compensar o movimento do pistão quando este se desloca (Compressão ou Exaustão dos gases).

As técnicas termográficas consistem na aplicação de tensões térmicas no objeto, medição da distribuição da temperatura da superfície e apresentação da mesma, de tal forma que as anomalias que representam as descontinuidades possam ser reconhecidas. 

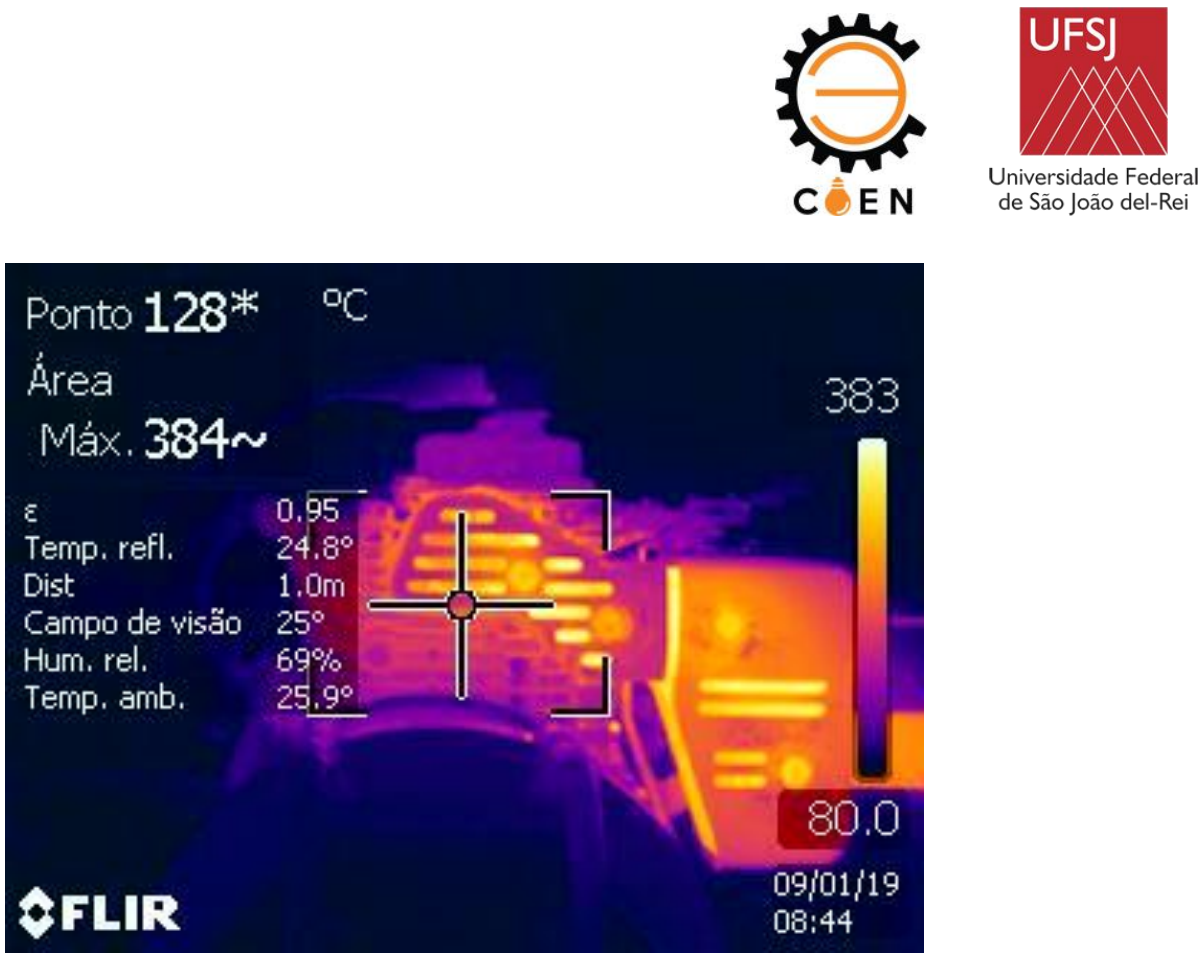

Figura 10. Termograma em condição ideal de funcionamento. Fonte: Autores.

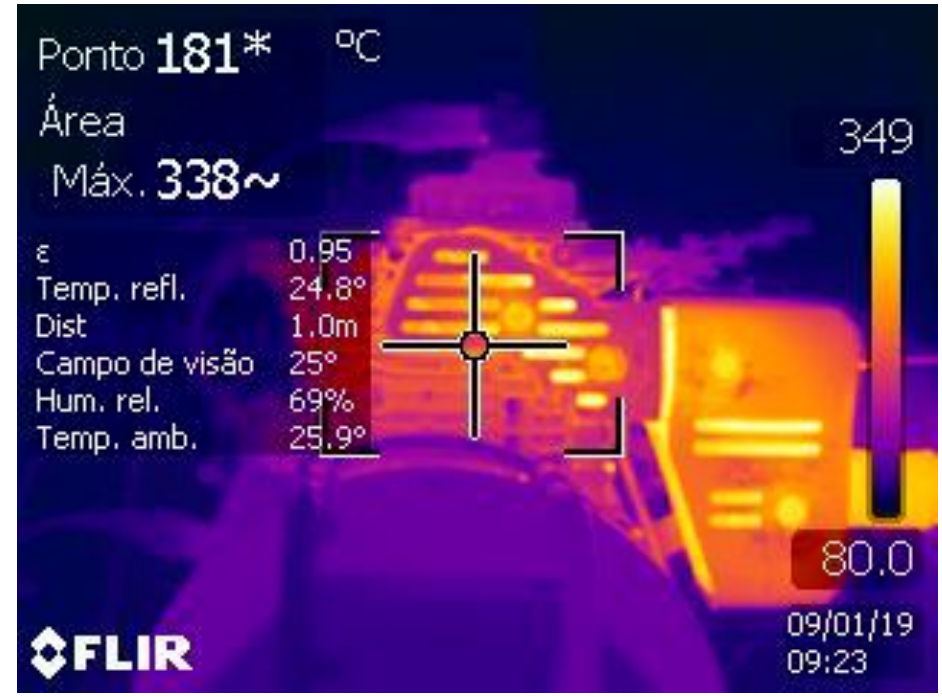

Figura 11. Termograma com desbalanceamento a $0^{\circ}$ do pistão em PMS. Fonte: Autores. 

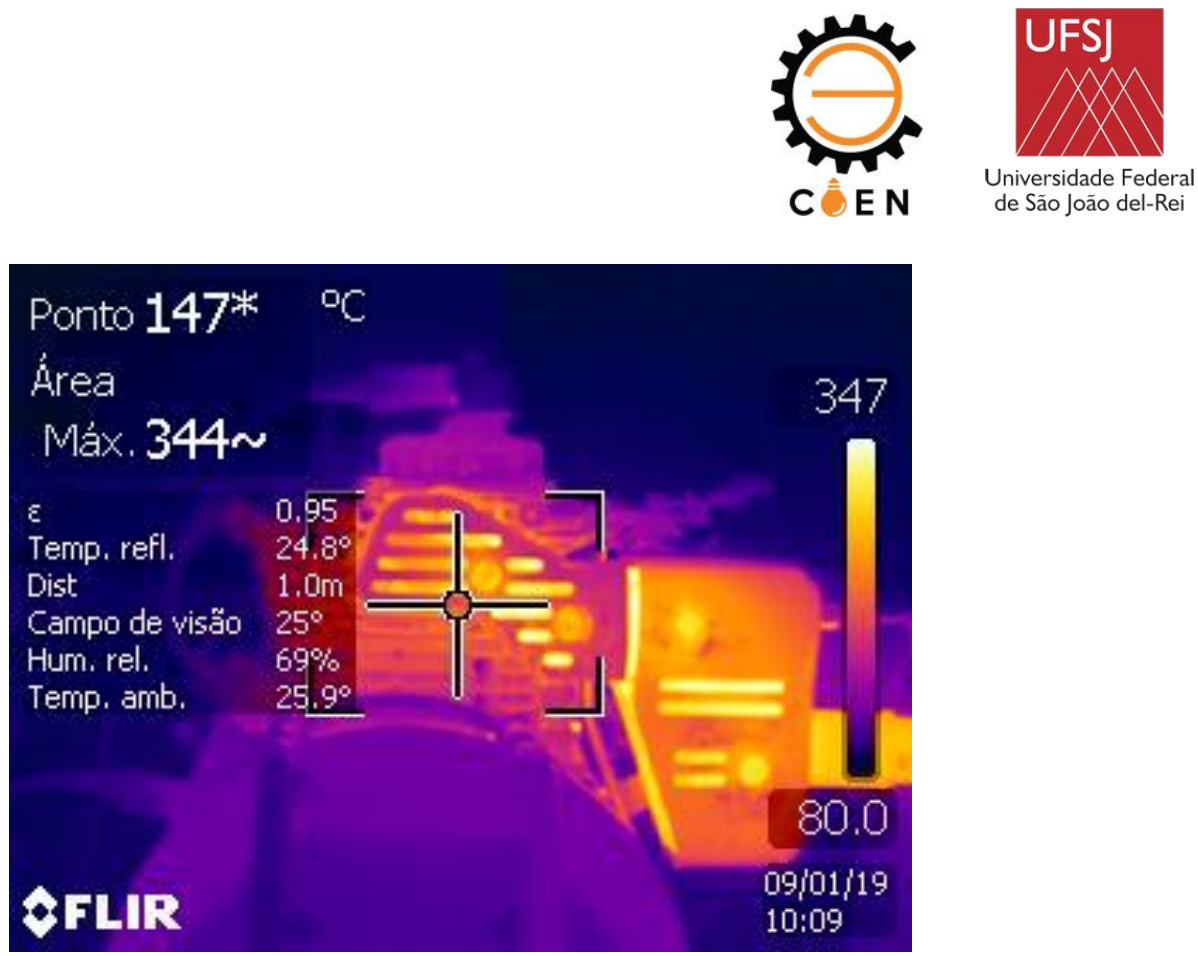

Figura 12. Termograma com desbalanceamento a $180^{\circ}$ do pistão em PMS. Fonte: Autores.

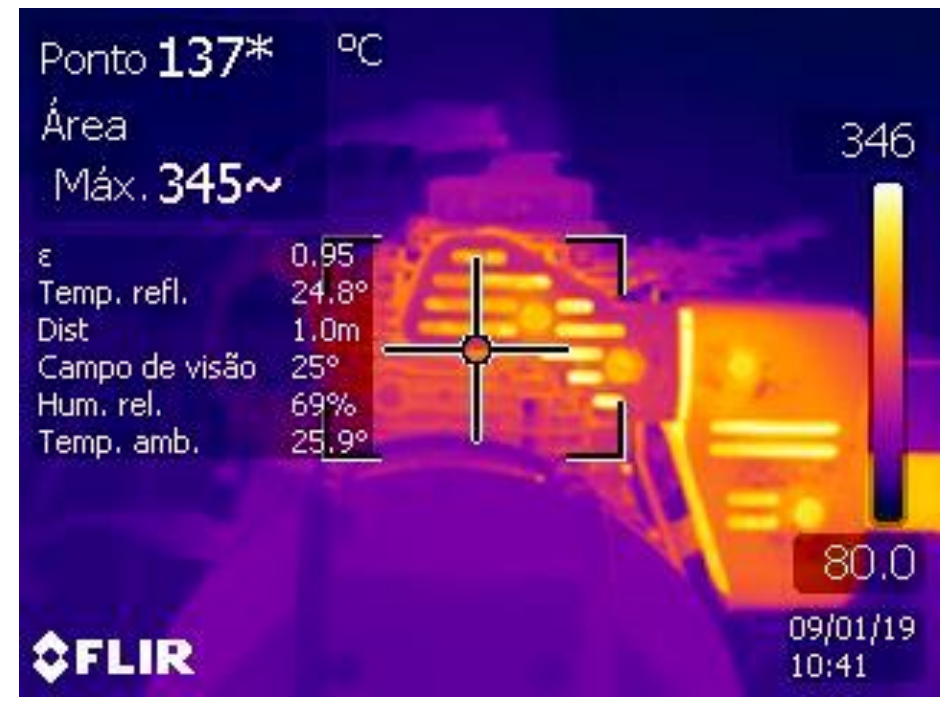

Figura 13. Termograma com desbalanceamento a $270^{\circ}$ do pistão em PMS. Fonte: Autores.

Por meio de testes não invasivos, com a câmera termográfica, foi possível observar a influência do desbalanceamento na variação da temperatura de trabalho do motor. A obtenção dos termogramas, para este trabalho, permite avaliar o aumento da temperatura na superfície do cilindro, cabeçote e escapamento, podendo assim caracterizar o aquecimento do motor e evitar que ele atinja temperaturas que possam causar falhas de origem térmica, como detonação e autoignição, e falhas de origem mecânica, como folga e desalinhamento, reduzindo assim o risco de uma parada abrupta.

Com base nos termogramas obtidos, é possível verificar que o desbalanceamento nas posições em que foi inserido contribuíram para a diminuição das temperaturas máximas de trabalho. Entretanto, levando-se em conta o ponto evidenciado nos termogramas, localizado na superfície do cilindro, nota-se que as temperaturas aumentaram consideravelmente para $0^{\circ}$ e apresentaram seu 

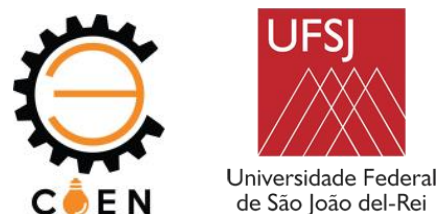

menor diferencial em $270^{\circ}$. Os resultados obtidos evidenciam a importância do correto balanceamento para o processo de combustão, pois ao ser desbalanceado o motor chegou a um diferencial de $50^{\circ} \mathrm{C}$ para um mesmo ponto, podendo representar gastos energéticos excessivos e ocasionar falhas como autoignição.

A caracterização térmica e vibracional de motores a combustão é de suma importância, visto que este tipo de estudo ainda é pouco abordado devido à complexidade de obtenção de parâmetros. Com este estudo, será possível acompanhar o desempenho do motor por meio de geração de espectros vibracionais e termogramas, que poderão predizer quando deverá ocorrer uma intervenção da manutenção.

\section{DIREITOS AUTORAIS.}

Os autores são os únicos responsáveis pelo conteúdo das informações contidas neste artigo.

\section{REFERENCIAS}

BACCARINI, L.M.R., Detecção e Diagnóstico de Falhas em Motores de Indução. Belo Horizonte: Universidade Federal de Minas Gerais, 179p. Tese (Doutorado), 2005.

BRITO, J. N. Desenvolvimento de um sistema inteligente híbrido para diagnóstico de falhas em motores de indução trifásicos. Campinas: Universidade Estadual de Campinas, 214p. Tese (Doutorado), 2002.

BASSI, Anderson Flávio. Análise da Perda energética no cilindro em motor diesel através da termografia. Dissertação de Pós-Graduação em Engenharia da Energia. Universidade Federal de São João Del Rei, 2012.

CAMPOS, Diogo Carlos Melo de Pinho. Implementação de técnicas e ferramentas para manutenção preditiva na Colep. Dissertação de Mestrado, Faculdade de Engenharia da Universidade do Porto, 2011.

ÇENGEL, Yunus A. Heat Transfer: A Practical Approach. 2 ed. McGraw-Hill, 2002.

DIONÍSIO, Gonçalo Marques. Proposta de um Sistema de Monitorização de Condição de Motores Diesel. Dissertação de Mestrado. Faculdade de Ciências e tecnologia Universidade Nova de Lisboa, 2017.

GOLDWINE, G., BOTTON, G., RIVIN, B., AND SHER, E., Assesment of the Quality of Combustion in Compression Ignition Engines through Vibration Signature Analysis, Fisita World Automotive Congress, Barcelona, 2004.

MALPICA, Luis Gustavo Torquatro. Manutenção preditiva de motores de combustão interna, à gasolina, através da técnica de análise de lubrificantes. 2007. 111 f. Dissertação (mestrado) - Universidade Estadual Paulista, Faculdade de Engenharia de Ilha Solteira, 2007.

OLIVEIRA, Davi Borges de. Manutenção preditiva; Termografia; Indústria sucroalcooleira; Manutenção preditiva; Termografia; Indústria sucroalcooleira. Trabalho de Conclusão de Curso apresentado ao Centro Universitário de Formiga - UNIFOR-MG, 2012.

PRADO JÚNIOR, Hudson Lobo do. Identificação de falhas em engrenagens na transmissão de trator agrícola por meio de análise de vibrações. Tese de Mestrado. Universidade Federal do Rio Grande do Sul, Pouso Alegre, 2003. 
REZENDE, J. C. F. Manutenção Preditiva em Turbomáquinas utilizando Sistemas Especialistas na Análise de Vibração. Tese de Mestrado. COPPE/UFRJ, Universidade Federal do Rio de Janeiro, Rio de Janeiro. 1991.

SOUZA, Valdir Cardoso de. Organização e gerenciamento da manutenção: programação e controle de manutenção / Valdir Cardoso de Souza. - $4^{\text {a }}$. Ed. - São Paulo: All Printe Editora. 2011.

\section{STUDY OF MOTOR THERMAL BEHAVIOR INTERNAL COMBUSTION SUBMITTED TO UNBALANCE IN DIFFERENT POSITIONS}

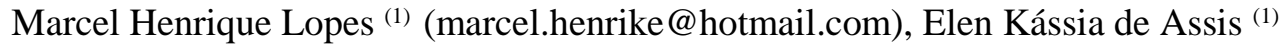
(elen.kassia@engenharia.ufjf.br), Jorge Nei Brito ${ }^{(2)}$ (brito@ufsj.edu.br), José Antônio da Silva ${ }^{(3)}$ (jant@ufsj.edu.br)

\footnotetext{
(1) Univ. Federal de São João Del Rei (UFSJ) - Grupo de Estudos e Pesquisas do Laboratório de Sistemas Dinâmicos (Gep_LASID)

(2) Universidade Federal de São João Del Rei (UFSJ) - Departamento de Engenharia Mecânica (DEMEC)

${ }^{(3)}$ Universidade Federal de São João Del Rei (UFSJ) - Departamento de Ciências Térmicas e dos Fluidos (DCTEF)
}

ABSTRACT: Unbalance is a factor that occurs frequently in equipment and to know how it presents itself in vibrational spectra and thermograms is of paramount importance to identify possible faults. The thermography, technique of analysis and characterization used in this work, is a technique of remote sensing that allows the measurement of temperatures and thermal imaging of equipment and / or processes, from the infrared radiation emitted naturally by the bodies. In electric motors, the way failure modes appear in spectra is known and consolidated. However, for internal combustion engines, this type of analysis is not so simple, making it necessary to adopt methods that allow non-invasive characterization of possible failure modes. For this study, the "Vibration Signature" of the motor was previously defined, which is the vibrational frequency of this in ideal operating conditions and its respective thermogram, so that the others were based on it. Then the unbalance masses were inserted so that the operant mode with this type of fault was characterized, thus allowing, in the event of unbalance, it could be identified by means of the vibrational data of the motor and its thermograms. The data were collected in three directions, vertical, horizontal and axial, through piezoelectric accelerometers that transmitted the signal to the online monitoring equipment IMx-S 16 of SKF. The vertical position presented the most interesting results for analysis. The data were stored and processed in SKF's @ ptitude Analyst software. The thermal images were obtained through the FLIR T200 camera and later processed in the FLIR Quick Report software.

KEYWORDS: Predictive maintenance. Thermography. Vibration. Internal combustion engine. Imbalance. 